\title{
LUPUS SCIENCE\& MEDICINE \\ Characteristics of azathioprine use and cessation in a longitudinal lupus cohort
}

\author{
Lucy Croyle, Alberta Hoi, Eric F Morand
}

To cite: Croyle L, Hoi A, Morand EF. Characteristics of azathioprine use and cessation in a longitudinal lupus cohort. Lupus Science \& Medicine 2015;2:e000105. doi:10.1136/lupus-2015000105

Received 25 May 2015 Revised 14 July 2015 Accepted 30 July 2015
CrossMark

\section{Centre for Inflammatory} Diseases, Monash University School of Clinical Sciences, Monash Medical Centre, Melbourne, Australia

Correspondence to Dr Lucy Croyle; lucy.croyle@monash.edu

\section{ABSTRACT}

Objective: Guidelines for azathioprine (AZA) use in systemic lupus erythematosus (SLE), including indications for initiation and cessation, are lacking. Clinical decision-making could be improved if reasons for cessation of AZA treatment were standardised. Methods: We determined the characteristics of AZA use in a cohort of patients with SLE and evaluated reasons for AZA cessation. Patients with SLE in a single centre had longitudinal recording of disease activity (Systemic Lupus Erythematosus Disease Activity Index 2000 (SLEDAI)-2k), laboratory investigations and treatment from 2007 to 2012.

Results: Of 183 patients studied, 67 used AZA on at least one occasion. There was no significant difference between AZA users and non-users in age or American College of Rheumatology criteria. Compared with those not treated with AZA, patients treated with AZA had higher disease activity (time-adjusted mean SLEDAI 5.2 \pm 0.3 vs $3.8 \pm 0.3, p=0.0028$ ) and damage (Systemic Lupus International Collaborating Clinics (SLICC)-SDI $1.6 \pm 0.3$ vs $1.2 \pm 0.1, p=0.0445$ ), and were more likely to have a positive dsDNA $(p=0.0130)$ and receive glucocorticoids $(p<0.0001)$. AZA therapy was ceased in $30 / 67(45 \%)$ patients. The predominant reasons for cessation were treatment de-escalation $14(47 \%)$, treatment failure $12(40 \%)$ and toxicity $3(10 \%)$. AZA was switched to mycophenolate mofetil (MMF) in 9/12 $(75 \%)$ of treatment failures, and this choice was strongly associated with active lupus nephritis.

Conclusions: AZA toxicity was uncommon, and many patients ceased therapy in the context of treatment deescalation. However, the frequent development of active lupus nephritis requiring MMF suggests the need to distinguish refractoriness, under-treatment and nonadherence to AZA in patients with SLE. These findings suggest that future studies of AZA metabolite measurement could prove valuable in the management of SLE.

\section{INTRODUCTION}

Azathioprine (AZA) is a valuable immunosuppressant for the treatment of multiple manifestations of systemic lupus erythematosus (SLE) ${ }^{1-5}$ Following the MAINTAIN trial $^{6}{ }^{7}$ and ALMS study, ${ }^{8}$ mycophenolate mofetil (MMF) has largely usurped AZA as the therapy of choice in lupus nephritis, for

\section{KEY MESSAGES}

- Azathioprine is a commonly used immunosuppressant for all manifestations of SLE, however indications for its use and cessation are not standardized.

- We describe the use and cessation of AZA in our SLE cohort, with $50 \%$ of cessation due to treatment failure or toxicity and the remainder undergoing de-escalation.

- Distinguishing true treatment refractoriness from undertreatment or non-compliance, and toxicity from disease activity may be enhanced with use of AZA metabolite measures.

both inducing remission and as maintenance therapy. However, in a patient population consisting predominantly of women in their child-bearing years, AZA is one of only a few medications deemed safe for use during pregnancy, ${ }^{9-11}$ and it remains widely used. Pre-therapy measurement of thiopurine methyltransferase (TPMT) enzyme levels is not universally implemented in rheumatology practice, and there is a lack of consensus about indications for initiation and cessation of AZA therapy in SLE, with no widely accepted treatment guidelines for its use. ${ }^{5}$ In addition, current weight-based dosing regimens for AZA fail to reflect inter-patient variability of AZA metabolism. ${ }^{12} 13$

Evidence in autoimmune hepatitis ${ }^{14}$ and inflammatory bowel disease (IBD) ${ }^{15}$ designates a target range for AZA metabolites that correlates with avoidance of serious toxicity, attaining and sustaining remission, as well as providing an indication of treatment adherence. ${ }^{16}$ This has not been applied in SLE. For patients with active SLE despite AZA, appropriate designation as non-compliant, treatment-refractory or undertreated could improve clinical decision-making as has been shown most clearly in IBD. ${ }^{15}$

Given the evolving prospects for improving clinical decision-making via therapeutic drug monitoring of AZA, we sought to evaluate patterns of AZA cessation in an SLE cohort, to determine whether reasons for cessation 
of AZA in routine practice supported the potential utility of metabolite monitoring. We found that a significant proportion of patients ceased AZA therapy due to poor control of disease, implying that designation of the cause of therapeutic failure through metabolite monitoring could frequently be of benefit.

\section{METHODS}

We performed a retrospective study of longitudinal data, acquired prospectively in a single centre. As previously described, ${ }^{17-19}$ all patients with SLE $(\geq 4$ American College of Rheumatology (ACR) criteria $^{20}$ ) attending the Lupus Clinic at Monash Medical Centre, Melbourne, Australia, were invited to be included in a database, approved by the Human Research Ethics committee of Monash Health. After written informed consent, baseline demographic and clinical data were collected on all patients during routine visits to the SLE clinic by the attending physician using standard proformas. Recording of disease activity (hybrid SELENA-Systemic Lupus Erythematosus Disease Activity Index 2000 $\left(\right.$ SLEDAI-2k) ${ }^{21}$ ), laboratory investigations (serum complement, anti-dsDNA antibodies, full blood count, electrolytes, liver function tests, proteinuria (urine protein: creatinine ratio) and urine microscopy) and treatment details are completed at each visit as previously described. ${ }^{17-19}$ Disease-related damage is recorded annually using the Systemic Lupus International Collaborating Clinics (SLICC)-SDI ${ }^{22}$ and time-adjusted mean SLEDAI (AMS), a measure of mean disease activity adjusted for the period of observation, calculated.

All patients included in the database between January 2007 and November 2012 were assessed for inclusion; data on medication use prior to enrolment in the database were not available. Inclusion criteria for this study were at least one complete set of visit data during the study period. Patients using AZA at any time during the period of observation were identified and details of disease activity and medication use extracted. AZA cessation was defined when documented use of AZA was terminated during the period of observation. Medications used prior to, during and following AZA use were documented, as were prednisolone dose changes at time of AZA cessation. Reasons for AZA cessation were designated as toxicity, treatment failure or de-escalation. Toxicity was defined when abnormal liver function or haematological parameters were present at the time of cessation. Treatment failure was defined where patients ceasing AZA underwent escalation of corticosteroid dose or institution of replacement immunosuppressive therapy. De-escalation was defined when AZA was ceased but no escalation in glucocorticoids or treatment replacement for AZA was instituted, assuming the definitions of toxicity or treatment failure were not met. Disease activity at the time of AZA cessation was evaluated to verify the presence of treatment failure.
Attainment of the recently described lupus low-disease activity state (LLDAS) ${ }^{23}$ at AZA cessation was assessed.

For comparison of means, continuous data were analysed using Student's t tests, while discontinuous data were analysed using Mann-Whitney rank tests. For comparisons of prevalence and calculation of ORs, Fisher's exact test was used. All statistical analyses were completed using GraphPad Prism Software V.6.0.

\section{RESULTS}

A total of 183 patients were studied. Characteristics of the study population are shown in table 1. Of 183 patients studied, 67 (37\%) used AZA on at least one occasion during the study period. Among patients who had used AZA, the median (range) dose of AZA used across the period of observation was $95 \mathrm{mg} /$ day (50-150 mg/day).

We next compared clinical characteristics between patients who used AZA and those who did not. As shown in table 2, there was no significant difference in age or number of ACR criteria fulfilled between patients using AZA and those not.

Patients using AZA had a significantly longer total period of observation, and a greater number of visits recorded than patients not using AZA (table 2). Patients who were treated with AZA at any time during the period of observation had significantly higher AMS, indicating higher disease activity across the period of observation ( $p=0.0028$, table 2). Ethnicity differed significantly, in that patients of Caucasian ethnicity were significantly over-represented among patients using AZA (table 2). No

\begin{tabular}{|c|c|}
\hline $\mathrm{N}$ & 183 \\
\hline Age (years, mean \pm SEM) & $44.4 \pm 15.0$ \\
\hline Ethnicity (Asian:Caucasian:other*) & $\begin{array}{l}90: 54: 39 \\
(49 \%: 30 \%: 21 \%)\end{array}$ \\
\hline Gender (female:male, n (\%)) & 154:29 (84\%:16\%) \\
\hline ACR criteria (median (range)) & $5(4-9)$ \\
\hline Duration SLE (years, mean \pm SEM) & $9.9 \pm 7.7$ \\
\hline Visits observed ( $\mathrm{n}$, median (IQR)) & $12(6-21)$ \\
\hline $\begin{array}{l}\text { Period observed (days, median } \\
\text { (IQR)) }\end{array}$ & $1113(469-1603)$ \\
\hline ANA positive (n (\%)) & $180(100 \%)$ \\
\hline ANA titre (median (range)) & $1280(80-2560)$ \\
\hline Anti-dsDNA positive (n (\%)) & $133(72.7 \%)$ \\
\hline Hypocomplementemia (n (\%)) & $139(75.9 \%)$ \\
\hline Use of prednisolone ( $\mathrm{n}(\%))$ & $138(75.4 \%)$ \\
\hline $\begin{array}{l}\text { Adjusted mean prednisone dose } \\
\text { (mg/day, mean } \pm \text { SEM) }\end{array}$ & $7.6 \pm 0.66$ \\
\hline AMS (mean \pm SEM) & $4.34 \pm 0.23$ \\
\hline SDI (median (range)) & $1(0-12)$ \\
\hline \multicolumn{2}{|c|}{$\begin{array}{l}\text { *Other-those identifying as 'other' or declining to be identified } \\
\text { ethnically. } \\
\text { ACR, American College of Rheumatology; AMS, (time) adjusted } \\
\text { mean SLEDAI; ANA, antinuclear antibody; SLE, systemic lupus } \\
\text { erythematosus; SLEDAI, Systemic Lupus Erythematosus Disease } \\
\text { Activity Index. }\end{array}$} \\
\hline
\end{tabular}


Table 2 Characteristics of azathioprine (AZA) users versus non-AZA users

\begin{tabular}{|c|c|c|c|}
\hline & AZA users & Non-AZA users & p Value \\
\hline $\mathrm{N}$ & 67 & 116 & \\
\hline Age (year, mean \pm SEM) & $43 \pm 2$ & $45 \pm 1$ & 0.4553 \\
\hline \multirow[t]{2}{*}{ Ethnicity (n; \%) (Asian:Caucasian:other ${ }^{\star}$ ) } & 29:32:6 & $61: 22: 33$ & $<0.0001$ \\
\hline & $43 \%: 47 \%: 10 \%$ & 53\%:19\%:28\% & \\
\hline ACR criteria (median (range)) & $5(4-9)$ & $5(4-9)$ & 0.5796 \\
\hline Duration SLE & $10.09 \pm 0.8840$ & $9.776 \pm 0.7368$ & 0.7906 \\
\hline Visits observed ( $\mathrm{n}$, median (IQR)) & $18(9-26)$ & $9(4-16)$ & $<0.0001$ \\
\hline Period observed (days, median (IQR)) & $1316(798-1701)$ & $889(369-1545)$ & 0.0094 \\
\hline Anti-dsDNA-positive & $54(80.6 \%)$ & $77(67.5 \%)$ & 0.0399 \\
\hline Hypocomplementemia (n (\%)) & $60(89.5 \%)$ & $79(68.1 \%)$ & 0.0011 \\
\hline AMS (mean \pm SEM) & $5.23 \pm 0.29$ & $3.82 \pm 0.31$ & 0.0028 \\
\hline SDI (median (range)) & $1(0-12)$ & $1(0-7)$ & 0.1422 \\
\hline Corticosteroid use & $65(97 \%)$ & $73(63 \%)$ & $<0.0001$ \\
\hline Adjusted mean prednisone dose (mg/day, mean \pm SEM) & $7.6 \pm 0.66$ & $5.6 \pm 0.62$ & 0.0386 \\
\hline Hydroxychloroquine use & $65 / 67(97 \%)$ & $104 / 116(89.7 \%)$ & NS \\
\hline
\end{tabular}

significant difference in cumulative damage, as measured using SLICC-SDI at the last assessment, was observed (table 2). Patients using AZA were more likely to have anti-dsDNA antibodies $(\mathrm{p}=0.0130)$ and to receive glucocorticoids ( $\mathrm{p}=0.0001$ ), and were exposed to a significantly higher adjusted mean glucocorticoid dose $(p=0.0386$, table 2), than patients not exposed to AZA. Concomitant treatment with hydroxychloroquine (HCQ) occurred in $65 / 67(97 \%)$ of the AZA cohort, and 104/116 (89.7\%) of non-AZA cohort (NS).

Of the 67 AZA users, 29 commenced AZA during the period of observation, allowing us a view of preceding medications and disease trajectory. Of those starting AZA during the period of observation, 24/29 had used preceding lupus medications including $\mathrm{MMF}$ (6 patients) methotrexate (MTX) (4), cyclophosphamide (2), rituximab (1), HCQ (22) and prednisolone (23). Thus, five patients commenced AZA de novo with no preceding medications during database capture.

We next assessed reasons for AZA cessation. Also, 30/ $67(45 \%)$ patients ceased AZA during the period observed, affording the opportunity to examine reasons for treatment cessation. Using the definitions outlined above, 14 patients (21\%) ceased AZA due to de-escalation, $12(18 \%)$ due to treatment failure and 3 (4\%) due to toxicity, with one patient unable to be classified. Among patients assessed as ceasing AZA due to de-escalation, 6/14 (43\%) met criteria for LLDAS, while $8 / 14(57 \%)$ did not. There was no difference in ethnicity among patients who did, or did not, cease AZA (not shown). As details of AZA treatment prior to enrolment in the database were not available, no analysis of treatment duration was undertaken.

Among 30 patients ceasing AZA, treatment failure was identified as the reason for cessation in $40 \%$. Treatment was changed to MMF in $9 / 12(75 \%)$ patients who ceased AZA due to treatment failure. Overall disease activity as measured by SLEDAI was numerically but not significantly higher in those in whom AZA was switched to MMF (AZA-MMF) compared with those who switched to other treatments (AZA-other) (table 3). None of the patients who ceased AZA due to treatment failure met criteria for LLDAS.

On the basis that MMF is most frequently used for the treatment of lupus nephritis, we analysed renal disease activity using the renal domains of SLEDAI (urinary casts, proteinuria, haematuria, pyuria). ${ }^{24}{ }^{25}$ AZA-MMF patients had significantly higher renal SLEDAI than AZA-other patients $(p=0.0214$, table 3$)$. Data on histological class of lupus nephritis are stored in our database; however, only patients with Class III/IV/V lupus nephritis qualify for MMF treatment in our centre. Contingency analysis revealed that the relative risk of active renal disease in AZA-MMF patients compared with AZA-other patients was $4.6(95 \%$ CI 1.1 to 18.4 , $\mathrm{p}=0.019$ ). This suggests that active lupus nephritis developing during AZA therapy was driving treatment escalation to MMF. Among the patients who switched from AZA to MMF, the median dose was $100 \mathrm{mg}$ /day (range $53-143$ ); a median (range) dose of $1.521 \mathrm{mg} / \mathrm{kg} /$ day (0.839-2.123). This was not different from the median (range) $1.527(0.547-2.50) \mathrm{mg} / \mathrm{kg} /$ day in the overall population on AZA. In contrast, lupus nephritis was not the major lesion in the 3/12 treatment failure patients whose therapy was changed to immunosuppressive treatment other than MMF.

Toxicity, in the form of leukocytopenia, was the reason for cessation in three patients ( $10 \%$ of patients ceasing AZA). Of the three neutropenic patients, a nadir of $1.3 \times 10^{9}$ leucocytes/L was observed in a patient with 
Table 3 Reasons for AZA cessation

\begin{tabular}{|c|c|c|c|c|}
\hline & $\begin{array}{l}\text { Total } \\
67 \\
\end{array}$ & $\begin{array}{l}\text { Cessation } \\
30\end{array}$ & $\begin{array}{l}\text { No cessation } \\
37\end{array}$ & \\
\hline De-escalation & & 14 & & \\
\hline Toxicity & & 3 & & \\
\hline \multirow[t]{2}{*}{ Treatment failure } & & 12 & & \\
\hline & & AZA-MMF* & AZA-other† & p Value \\
\hline$(n,(\%))$ & & $9(75 \%)$ & $3(25 \%)$ & \\
\hline SLEDAI (mean \pm SEM) & & $10.14 \pm 2.45$ & $5.59 \pm 1.2$ & NS \\
\hline Renal SLEDAI (mean \pm SEM) & & $4.44 \pm 1.0$ & $1.52 \pm 0.65$ & 0.0214 \\
\hline
\end{tabular}

likely lupus-induced neutropenia, evidenced by further reduction to $0.48 \times 10^{9}$ leucocytes/L 14 months post cessation of AZA, with no other cytopenia-inducing medications. The other two patients had neutrophil nadirs of 0.97 and $1.78 \times 10^{9}$ leucocytes/L, both of which improved over 1-4 months with cessation of AZA and use of prednisolone, consistent with AZA-mediated bone marrow suppression.

\section{DISCUSSION}

In SLE, AZA is generally prescribed for active non-renal disease, using weight-based dosing with varying suggested adjustments for renal impairment. ${ }^{26}{ }^{27}$ As control of disease activity is a key goal in SLE, ${ }^{28}{ }^{29}$ the presence of active disease despite AZA therapy may result in the presumption of inefficacy and switching of AZA to another therapy. Dose escalation is an alternative to switching, but the frequency of dose-limiting AZA toxicity in patients with SLE is not well described. An individualised metabolite monitoring-based approach to AZA therapy in SLE has been suggested as a way to distinguish disease activity due to underexposure or nonadherence as opposed to medication refractoriness, and potentially distinguish AZA toxicity from active SLE as a cause of leucopenia. ${ }^{30}$ However, the frequency of these differing outcomes in AZA-treated patients with SLE is poorly understood, and hence the potential benefit of the application of AZA metabolite monitoring as a standard of care in SLE is unclear. We therefore undertook a study of AZA use in a longitudinally followed SLE cohort, focusing on reasons for treatment cessation, to determine whether reasons for AZA termination in routine practice supported the potential use of monitoring.

First, we observed that AZA use was common. In this cohort, AZA was prescribed in patients with more severe disease, as suggested by higher disease activity, anti-dsDNA positivity and use of corticosteroids. An average AMS of $>5$ indicates inadequate overall disease control in AZA-treated patients. This finding alone suggests the need for improved use of AZA in SLE, potentially using AZA metabolite monitoring to optimise dosing.

Second, a significant proportion of patients ceased AZA therapy during the period of observation. The most common reason for AZA cessation was treatment de-escalation, followed by treatment failure. In contrast, cessation of AZA due to toxicity was uncommon. Nearly a quarter of all patients with SLE treated with AZA ceased therapy in a setting of treatment de-escalation. Long-term continuation of HCQ is now commonplace, ${ }^{31}$ but there is a lack of data regarding the optimal duration of other therapies, especially in the setting of lowdisease activity. ${ }^{3}$ Interestingly, not all patients who ceased AZA for treatment de-escalation had 'low-disease activity' according to a definition proposed recently. ${ }^{23}$ The current study was not designed to assess outcomes after treatment de-escalation, but it would be useful in future studies to investigate outcomes subsequent to de-escalation of AZA therapy in patients with stable disease.

Outside the setting of clinical trials, treatment cessation decisions are based on a myriad of factors, and while many are captured in longitudinal cohort databases such as that studied here, others, including the impact of individual patient preference, and subjective measures of intolerability (nausea) are not. ${ }^{32}$ This highlights a limitation of the current study, in that the data were originally collected for the purpose of analysing longitudinal disease activity ${ }^{17-19}$ and were retrospectively analysed. Further limitations include the potential bias arising from studying a single-centre cohort. Finally, compliance and appropriate documentation cannot be assessed in this format.

The second most frequent reason for AZA cessation was treatment failure, defined as an escalation of therapy in the setting of active disease. There is increasing acceptance of the use of MMF for the treatment of lupus nephritis, ${ }^{8}$ and active nephritis was the dominant clinical feature in patients changing from AZA to MMF in the current study. The observation that 9 of 67 
AZA-treated patients developed active lupus nephritis requiring escalation to MMF suggests these cases were regarded by the treating physician as AZA-refractory. While AZA dosing recommendations vary according to disease manifestations of SLE, a maximum dose of $2 \mathrm{mg} / \mathrm{kg}$ is advised as maintenance therapy for lupus nephritis. ${ }^{36}$ The median dose in AZA-MMF patients was $1.5 \mathrm{mg} / \mathrm{kg} /$ day, and only two patients who transitioned to MMF in the setting of active renal disease were receiving a dose $\geq 2 \mathrm{mg} / \mathrm{kg}$, suggesting these patients may have been undertreated.

We propose that distinguishing between true treatment refractoriness, as opposed to under-dosing and nonadherence, and between toxicity and lupus-related leucopenia, would be possible through the measurement of AZA metabolites. Half of all patients on AZA in this study ceased treatment due to presumed inefficacy or toxicity, suggesting that as many as half of all patients with SLE treated with AZA could benefit from metabolite monitoring. One study in IBD using an AZA metabolite-based dosing algorithm found $24 \%$ of patients avoided inappropriate dose escalation, and $87 \%$ of patients achieved improved clinical outcomes. ${ }^{15}$ The application of such an approach in SLE has been suggested, ${ }^{30}$ but application of such an approach is limited by the fact that an empirically defined therapeutic range for AZA metabolites in SLE is lacking. Two small studies in mixed cohorts of connective tissue disease patients confirmed high interindividual variation of metabolite levels, and poor correlation of metabolite levels with weight-adjusted doses. ${ }^{12} 33$ A 6-month dose escalation study of 50 patients with SLE identified a target level of AZA metabolites that correlated with efficacy. ${ }^{34}$ Doses required to achieve this were approximately $3 \mathrm{mg} / \mathrm{kg} /$ day, higher than current dosing guidelines for lupus nephritis maintenance ${ }^{3}$ and similar to maximum dosing recommendations for other autoimmune manifestations. ${ }^{35} 36$ This suggests that inappropriate designation of patients as AZA-refractory may be common in SLE.

Pre-drug TPMT testing was not routinely undertaken in our cohort. It remains a matter of debate whether genotype or phenotype (TPMT enzyme testing) is superior in SLE. ${ }^{37}$ The observation that TPMT levels may not always determine potential marrow suppression ${ }^{39}$ nor individual dose requirements ${ }^{40}$ promotes ongoing conjecture regarding its utility. TPMT measurements potentially provide pretreatment information regarding potential risk of bone marrow suppression, whereas measurement of AZA metabolites can provide a real-time representation of an individual's handling of AZA. ${ }^{41}$

In this study, cessation of AZA treatment for toxicity was distinctly uncommon. Insufficient data preclude definitive attribution of the three cases of cytopenia to drug toxicity or disease activity. The ability to distinguish between drug toxicity and disease activity in patients with cytopenia during AZA therapy has potential to inform treatment choices and would also be assisted by metabolite measurement. The fourfold higher prevalence of treatment cessation for treatment failure than for toxicity implies that current focus of laboratory testing on adverse effects of AZA may be less valuable to clinicians than metabolite measurement enabling dose optimisation and hence disease control.

In conclusion, AZA was a frequently used immunosuppressant in this SLE cohort, but was withdrawn in a high proportion of cases. AZA cessation for toxicity was infrequent, while development of active lupus, including nephritis, requiring treatment escalation to mycophenolate was much more frequent. This suggests that breakthrough disease activity, implying AZA-refractoriness, undertreatment or non-adherence, greatly exceeds AZA toxicity as a management issue in SLE. In addition, when combined, the potential for AZA metabolite monitoring to influence physician decisions was present in half of all patients with SLE treated with AZA. We conclude that knowledge of AZA metabolite concentrations could potentially aid decision-making as part of the standard of care in SLE, for which prospective studies of AZA metabolite levels in SLE and strategy trials of recently proposed metabolite-based dosing algorithms ${ }^{30}$ are required. The outcomes of such research could include more stringent guidelines for the use of AZA in SLE, potentially resulting in highly cost-effective reductions in disease activity and prevention of treatment escalation.

Acknowledgements Susan Morton, Research Nurse, Monash Medical Centre, Melbourne, Australia.

Contributors All authors have contributed to the article's evolution, with majority of work being done by LC, with assistance in the form of editing, format and statistics from EFM, and editing and database assistance from $\mathrm{AH}$.

Funding Australian Rheumatology Association-the SA LSS Support Group funded by Arthritis South Australia.

Competing interests None declared.

Provenance and peer review Not commissioned; externally peer reviewed.

Data sharing statement There are no data sharing instructions or arrangements with no further unpublished data.

Open Access This is an Open Access article distributed in accordance with the Creative Commons Attribution Non Commercial (CC BY-NC 4.0) license, which permits others to distribute, remix, adapt, build upon this work noncommercially, and license their derivative works on different terms, provided the original work is properly cited and the use is non-commercial. See: http:// creativecommons.org/licenses/by-nc/4.0/

\section{REFERENCES}

1. Okon LG, Werth VP. Cutaneous lupus erythematosus: diagnosis and treatment. Best Pract Res Clin Rheumatol 2013;27:391-404.

2. Janssens P, Arnaud L, Galicier L, et al. Lupus enteritis: from clinical findings to therapeutic management. Orphanet J Rare Dis 2013;8:67.

3. American College of Rheumatology guidelines for screening, treatment, and management of lupus nephritis. 2012:797-808.

4. Bertsias G, loannidis JPA, Boletis J, et al. EULAR recommendations for the management of systemic lupus erythematosus. Report of a Task Force of the EULAR Standing Committee for International Clinical Studies Including Therapeutics; 2008:195-205.

5. Pego-Reigosa JM, Cobo-lbáñez T, Calvo-Alén J, et al. Efficacy and safety of nonbiologic immunosuppressants in the treatment of nonrenal systemic lupus erythematosus: a systematic review. Arthritis Care Res 2013;65:1775-85. 
6. Houssiau FA, D'Cruz D, Sangle S, et al. Azathioprine versus mycophenolate mofetil for long-term immunosuppression in lupus nephritis: results from the MAINTAIN Nephritis Trial. Ann Rheum Dis 2010;69:2083-9.

7. Morris HK, Canetta PA, Appel GB. Impact of the ALMS and MAINTAIN trials on the management of lupus nephritis. Nephrol Dial Transplant 2013:28:1371-6.

8. Isenberg D, Appel GB, Contreras G, et al. Influence of race/ethnicity on response to lupus nephritis treatment: the ALMS study. Rheumatol 2010;49:128-40.

9. Berden JHM. Azathioprine, the Cinderella in the treatment of lupus nephritis. Nephrol Dial Transplant 2009;24:2611-12.

10. Fischer-Betz R, Specker C, Brinks R, et al. Low risk of renal flares and negative outcomes in women with lupus nephritis conceiving after switching from mycophenolate mofetil to azathioprine. Rheumatol 2013;52:1070-6.

11. Østensen M, Khamashta M, Lockshin M, et al. Anti-inflammatory and immunosuppressive drugs and reproduction. Arthritis Res Ther 2006;8:209.

12. Schmiegelow K, Kriegbaum NJ. 6-Thioguanine nucleotide accumulation in erythrocytes during azathioprine treatment for systemic connective tissue diseases: a possible index for monitoring treatment. Ann Rheum Dis 1993:52:152-4.

13. Bradford K, Shih DQ. Optimizing 6-mercaptopurine and azathioprine therapy in the management of inflammatory bowel disease. World $J$ Gastroenterol 2011:17:4166-73.

14. Dhaliwal HK, Anderson R, Thornhill EL, et al. Clinical significance of azathioprine metabolites for the maintenance of remission in autoimmune hepatitis. Hepatology 2012;56:1401-8.

15. Haines ML, Ajlouni Y, Irving PM, et al. Clinical usefulness of therapeutic drug monitoring of thiopurines in patients with inadequately controlled inflammatory bowel disease. Inflamm Bowel Dis 2011;17:1301-7.

16. Stocco G, Londero M, Campanozzi A, et al. Usefulness of the measurement of azathioprine metabolites in the assessment of non-adherence. J Crohns Colitis 2010;4:599-602.

17. Golder V, Connelly K, Staples M, et al. Association of Asian ethnicity with disease activity in SLE: an observational study from the Monash Lupus Clinic. Lupus 2013;22:1425-30.

18. Vincent FB, Northcott M, Hoi A, et al. Clinical associations of serum interleukin-17 in systemic lupus erythematosus. Arthritis Res Ther 2013;15:R97.

19. Vincent FB, Northcott $M$, Hoi A, et al. Association of serum B cell activating factor from the tumour necrosis factor family (BAFF) and a proliferation-inducing ligand (APRIL) with central nervous system and renal disease in systemic lupus erythematosus. Lupus 2013;22:873-84.

20. Tan EM, Cohen AS, Fries JF, et al. The 1982 revised criteria for the classification of systemic lupus erythematosus. Arthritis Rheum 1982;25:1271-7.

21. Buyon JP, Petri MA, Kim MY, et al. The effect of combined estrogen and progesterone hormone replacement therapy on disease activity in systemic lupus erythematosus: a randomized trial. Ann Intern Med 2005;142:953-62.

22. Stoll T, Seifert B, Isenberg DA. SLICC/ACR Damage Index is valid, and renal and pulmonary organ scores are predictors of severe outcome in patients with systemic lupus erythematosus. $\mathrm{Br}$ Rheumatol 1996;35:248-54.

23. Lau CS, Nikpour M, Navarra SV, et al. Consensus definition of a low disease activity state in systemic lupus erythematosus. Ann Rheum Dis 2014;72:A267-7.
24. Bombardier C, Gladman DD, Urowitz MB, et al. Derivation of the SLEDAI. A disease activity index for lupus patients. Arthritis Rheum 1992;35:630-40.

25. Yee C-S, Farewell VT, Isenberg DA, et al. The use of Systemic Lupus Erythematosus Disease Activity Index-2000 to define active disease and minimal clinically meaningful change based on data from a large cohort of systemic lupus erythematosus patients. Rheumatology 2011;50:982-8.

26. Aronoff GR, Bennett WM, Berns JS, et al. eds. Drug prescribing in renal failure: dosing guidelines for adults. 5th edn. Philadelphia: American College of Physicians, 2007:97,177

27. van Laar JM. Immunosuppressive drugs. In: Firestein GS, Budd RC, Gabriel SE, et al. eds. Kelley's textbook of rheumatology. 9th edn. Saunders, 2013:946-8.

28. Franklyn K, Hoi A, Nikpour M, et al. The need to define treatment goals for systemic lupus erythematosus. Nat Rev Rheumatol 2014;10:567-71.

29. Korbet SM, Lewis EJ, Collaborative Study Group. Severe lupus nephritis: the predictive value of $a \geq 50 \%$ reduction in proteinuria at 6 months. Nephrol Dial Transplant 2013;28:2313-18.

30. Croyle L, Morand EF. Optimising the use of existing therapies in lupus. Int J Rheum Dis 2015;18:129-37.

31. Costedoat-Chalumeau N, Amoura Z, Hulot J-S, et al. Low blood concentration of hydroxychloroquine is a marker for and predictor of disease exacerbations in patients with systemic lupus erythematosus. Arthritis Rheum 2006:54:3284-90.

32. Thompson AJ, Newman WG, Elliott RA, et al. The cost-effectiveness of a pharmacogenetic test. A trial-based evaluation of TPMT genotyping for azathioprine. Value Health 2014;17:22-33.

33. Schedel J, Gödde A, Schütz E, et al. Impact of thiopurine methyltransferase activity and 6-thioguanine nucleotide concentrations in patients with chronic inflammatory diseases. Ann N Y Acad Sci 2006;1069:477-91.

34. Askanase AD, Wallace DJ, Weisman $\mathrm{MH}$, et al. Use of pharmacogenetics, enzymatic phenotyping, and metabolite monitoring to guide treatment with azathioprine in patients with systemic lupus erythematosus. J Rheumatol 2009;36:89-95.

35. Meggitt SJ, Anstey AV, Mohd Mustapa MF, et al. British Association of Dermatologists' guidelines for the safe and effective prescribing of azathioprine 2011. Br J Dermatol 2011;165:711-34.

36. Friedman $A B$, Sparrow MP, Gibson PR. The role of thiopurine metabolites in inflammatory bowel disease and rheumatological disorders. Int J Rheum Dis 2014;17:132-41.

37. Chouchana L, Narjoz C, Loriot M-A. TPMT status determination: the simplest is the most effective? J Crohns Colitis 2012;6:807-8.

38. Booth RA, Ansari MT, Tricco AC, et al. Assessment of thiopurine methyltransferase activity in patients prescribed azathioprine or other thiopurine-based drugs. Agency for Healthcare Research and Quality; 2010;1-282. Evidence Report/Technology Assessment No.196.

39. Naughton MA, Battaglia E, O'Brien S, et al. Identification of thiopurine methyltransferase (TPMT) polymorphisms cannot predict myelosuppression in systemic lupus erythematosus patients taking azathioprine. Rheumatology 1999;38:640-4.

40. Chen D, Lian F, Yuan S, et al. Association of thiopurine methyltransferase status with azathioprine side effects in Chinese patients with systemic lupus erythematosus. Clin Rheumatol 2013;33:499-503.

41. Wang L, Weinshilboum R. Thiopurine S-methyltransferase pharmacogenetics: insights, challenges and future directions. Oncogene 2003;25:1629-38. 